\title{
Article \\ Optimization of water distribution networks using Genetic Algorithm based SOP-WDN program
}

\author{
Uchit Sangroula 1, Kuk-Heon Han 2, Kang-Min Koo ${ }^{1}$, Kapil Gnawali ${ }^{1}$, and Kyung-Taek Yum 1, * \\ 1 Graduate School of Water Resources, Sungkyunkwan University, Suwon 16419, Korea; uchit@skku.edu; \\ koo00v@skku.edu; kapil@g.skku.edu \\ 2 Smart Water Technology and Consulting, Ltd., Incheon 21315, Korea; kuk0904@skku.edu \\ * Correspondence: kwfyum@skku.edu; Tel.: +82-31-290-7645
}

\begin{abstract}
Water distribution networks are vital infrastructure, needed for providing consumers with sufficient water of appropriate quality. The cost of construction, operation, and maintenance of such networks is extremely large. The problem of optimization of a water distribution network is governed by the type of water distribution network and the size of pipelines placed in the distribution network. This problem of the optimal diameter allocation of pipes in a distribution network has been heavily researched over the past few decades. This study describes the development of a computer program, 'Smart Optimization Program for Water Distribution Networks' (SOP-WDN), which applies Genetic Algorithm to the problem of the least-cost design of water distribution networks. SOP-WDN demonstrates the application of an evolutionary optimization technique, Genetic Algorithm, linked with a hydraulic simulation solver EPANET, for the optimal design of water distribution networks. The developed program was applied to three benchmark water distribution network optimization problems and produced consistently good results. SOP-WDN can be utilized as a tool for guiding engineers during the design and rehabilitation of water distribution pipelines.
\end{abstract}

Keywords: Water distribution networks, Optimization, Genetic Algorithm, EPANET

\section{Introduction}

A Water Distribution Network (WDN) is comprised of various elements, such as reservoirs, pumps, pipes, tanks, and valves. Around $80 \%$ of the total cost of a water supply project is invested in its water distribution system [1]. Hence the design of a cost-effective and reliable water distribution network is a must. Optimization of the WDN involves the design of a reliable, efficient, and cost-effective distribution network that fulfils the necessary water demands, while maintaining adequate pressure heads.

Over the years, numerous researchers have presented many different methods for obtaining the optimal solution to the pipe network optimization problem. The Hardy cross method is considered as the oldest method for solving a pipe network. In this method, at any pipe junction, the algebraic sum of flow must be zero, and the algebraic sum of pressure drops at any loop must also be zero [2]. This method was improved upon by many other researchers. Alperovits and Shamir [3] proposed one of the most significant approaches for solving the problem of water distribution network design by utilizing the successive Linear Programming Gradient (LPG) method. This method was adopted and further expanded upon by other researchers $[4,5]$.

However, deterministic methods, such as linear programming and non-linear programming, presented drawbacks, such as entrapment in local minima, and dependence on the starting point. Hence, they failed to obtain near optimal solutions for complex, multi-objective, real-world pipe network problems. To overcome these drawbacks, researchers began to utilize meta-heuristic algorithms (Genetic Algorithms, Simulated Annealing, etc.) for water network design problems. These techniques include algorithms 
having some stochastic components. Goldberg and Kuo introduced stochastic methods for the optimization of water distribution networks using the principles of natural selection and genetics [6]. Simpson et al. used simple Genetic Algorithms (GA), and obtained near optimal solution [7], while Simpson et al. [8] compared the GA technique with other methods, such as complete enumeration and non-linear optimization, and concluded that the GA technique generates multiple alternative solutions that are both practical and close to the optimum. The results obtained by Ref. [7] were further improved upon by Dandy et al. [9] using the concept of variable power scaling of the fitness function, an adjacency mutation operator, and gray codes. Savic and Walters developed the computer model GANET [10] that utilizes GA for the least-cost design of pipe networks.

To avoid unfeasible solutions due to the violation of constraints, a penalty factor is necessary during the selection process of GA. Deb and Agrawal [11] developed a nichedpenalty method to more effectively solve constrained optimization problems using GAs. $\mathrm{Wu}$ and Simpson [12] demonstrated significant improvements in efficiency and robustness for single-objective optimization utilizing a boundary search method. Liong and Atiquzzaman used the Shuffled Complex Evolution (SCE) linked with EPANET hydraulic network solver [13] to obtain the least cost of some well-known water distribution networks in the literature. SCE was demonstrated to be a potential alternative to other optimization algorithms, due to its faster computational speed. Other algorithms, like the Shuffled Frog-Leaping Algorithm (SFLA) by Eusuff [14] and Harmony Search Algorithm (HS) by Geem [15], have obtained comparable results, and proven to be effective tools for the optimal design of water networks.

Some studies consider a single economic objective (least-cost) to formulate the network optimization and rehabilitation problem, whereas others consider a multi-objective optimization approach that compares interesting trade-offs (e.g., a slight pressure deficit can sometimes be outweighed by substantial cost reduction) [16]. To improve network reliability, Chandramouli and Malleswararao [17] used fuzzy logic based on the excess pressure available at demand nodes. Jin et al. analyzed additional objectives, like considering both pressure and velocity violations [18]. More recent developments include improving algorithm convergence by using an engineered initial population, rather than a random one [19], and improvement of computational efficiency via the reduction of search space [20].

\subsection{Problem Formulation}

Cost-effective WDN design is a discrete optimization problem, as the individual pipe sizes are to be selected from a list of available commercial size diameters. The search space can be determined as the number of available diameters, raised to the power of the number of pipes in the network [21]; e.g., if 8 different commercial pipe sizes are available for the design of a WDN having 10 pipelines, the search space size would be $8^{10}$, i.e., $1,073,741,824$ different pipe combinations. Hence, even for a relatively small pipe network, the search space is large. The design of an economically optimal water distribution network is a difficult task, because it involves solving many complex, non-linear, and discontinuous hydraulic equations, while simultaneously optimizing pipe sizes and other network components [22, 23].

Optimization of a water distribution network aims to find the optimal pipe diameters in the network for the given layout and demand requirements. The optimal pipe sizes that satisfy all implicit constraints (conservations of mass and energy), and explicit constraints (hydraulic and design constraints) are selected in the final network.

The continuity equation is given as:

$$
\sum_{i=1}^{n} q_{i}=0
$$


The continuity equation is applied to each node, with $q_{i}$ being the flow rate (flow into and flow out of the node), and $n$ the number of pipes connected at the node.

The energy equation is given as:

$$
\sum_{i=1}^{m} h_{i}=0
$$

The energy equation is applied to each loop in the distribution network, where $h_{i}$ is the head loss in each pipe, and $m$ the number of pipes in the loop.

The objective function is the total cost of the given network. The total cost CT is calculated as:

$$
C_{T}=\sum_{i=1}^{N_{p}} C_{i}\left(D_{i}\right) \cdot L_{i}
$$

where, $N_{P}$ is the total number of pipes, $C_{i}\left(D_{i}\right)$ the cost per unit length of pipe $i$ with diameter $D_{i}$, and $L_{i}$ the length of pipe $i$. The objective function is to be minimized under the implicit constraints and explicit constraints.

The head loss is the sum of the local head losses and the friction head losses. The equation used to calculate the head loss is the Hazen-Williams (H-W) equation:

$$
h_{f}=4.72 C^{-1.85} Q^{1.85} D^{-4.87} L
$$

where, $h_{f}$ is the head loss, $Q$ the flow rate, $C$ the Hazen-Williams coefficient, $D$ the pipe inside diameter, and $L$ the pipe length.

\section{Materials and Methods}

\subsection{Genetic Algorithm}

Genetic Algorithm (GA) is a search algorithm based on the mechanics of natural selection and natural genetics [24]. Although stochastic at certain aspects, genetic algorithm is not entirely random, as it utilizes historical information to determine new search points. GA has been widely utilized to solve optimization problems in multiple fields [25]. Following the concept of 'survival of the fittest', improvements in solutions evolve from past generations, until a near optimal solution is obtained. In Genetic Algorithm, the candidate solutions are represented by chromosomes (e.g., binary strings), and are collectively known as the population. The chromosomes are then evolved in each subsequent generation, according to their fitness. The fitness evaluation of each candidate solution depends upon how well it the meets the requirements of a pre-defined objective function (e.g., lowest cost). The fitter the candidate solution, the more probability it will have of being selected for reproduction. Hence, the fitter chromosomes replace the less fit chromosomes, and the process continues until a near optimal solution is found.

The general idea of GA in a pipe network optimization problem is to select a population of initial solution points, scattered randomly in the optimization space, and then converge iteratively to better solutions, until the desired criteria for stopping are achieved. The steps for using GA for pipe network optimization can be briefly described as follows [7]:

1. Generation of initial population

The GA randomly generates an initial population of coded strings (binary) representing pipe network solutions of population size N. Each of the N strings represents a possible combination of pipe sizes.

2. Computation of network cost 
For each $\mathrm{N}$ string in the population, the GA decodes each substring into the corresponding pipe size, and computes the total network cost (material cost, construction cost, etc.).

3. Hydraulic analysis of each network

A steady state hydraulic network solver computes the heads and discharges under the specified demand patterns for each of the network designs in the population. The actual nodal pressures are compared with the minimum allowable pressure heads, and any pressure deficits are noted. Similarly, maximum allowable velocities and velocity defects in the network are also noted.

4. Computation of penalty cost

The GA assigns a penalty cost for each individual network design in the population if a pipe network does not satisfy the pressure and velocity constraints (for example, pressure violation at a particular node if the pressure in the node is less or greater than the desired pressure).

5. Computation of total network cost

The total cost of each network in the current population is then taken as the sum of the network cost (Step 2) and the penalty cost (Step 4).

6. Computation of the fitness

The fitness of the coded string is taken as some function of the total network cost. For each proposed pipe network in the current population, fitness can be computed as the inverse or the negative value of the total network cost (Step 5).

7. Generation of a new population using the selection operator

The GA generates new members for the next generation by a selection scheme that depends on the fitness of the initial members.

8. The crossover operator

Crossover occurs with some specified probability for each pair of parent strings selected in Step 7. A uniform type of crossover operator is commonly used to accompany the comparatively large string size for pipe network optimization.

9. The mutation operator

Mutation occurs with some specified probability of mutation for each bit in the strings that have undergone crossover. The purpose of mutation operator is to maintain genetic diversity from one generation of a population to another.

10. Production of successive generations

The use of the three operators described above produces a new generation of pipe network designs using Steps 2 to 9. The GA repeats the process to generate successive generations. The final costs and pipe network designs are stored, and as cheaper cost alternatives that meet the required constrains are generated, updated.

\subsection{EPANET}

EPANET is a computer program that can perform extended hydraulic and water quality simulations for pressurized water distribution networks (Rossman, [26]). Generally, a water distribution network consists of many elements, such as pipes (links), pipe junctions (nodes), pumps, control valves, and tanks/reservoirs. EPANET solves the water distribution network for the flow of water in each pipe, pressure at each junction, water height in each tank, concentration of chemical species, etc. During the hydraulic analysis of the water distribution network, EPANET solves both the conservation of mass and energy equations.

EPANET-MATLAB Toolkit is an open-source software for interfacing a drinking water distribution system simulation library, EPANET, with the MATLAB technical computing language developed by Eliades [27]. The Toolkit allows users to access EPANET and EPANET-MSX through their shared object libraries, as well as their executables. EPANET can be called and used through a programming interface by an external software, which can be written in different programming languages (such as $\mathrm{C} / \mathrm{C}++$, Python, or MATLAB). 
Generally, a large number of commands have to be written to achieve specific results, such as extracting the node pressures, pipe diameters, pipe roughness coefficients, or specifying demand patterns. However, in the EPANET-MATLAB toolkit, a significant part of the repetitive code is already included in the toolkit functions, and can be used directly.

\subsection{SOP-WDN}

Smart Optimization Program for Water Distribution Networks (SOP-WDN) is a computer program that has been developed by Smart Water Grid (SWG) research works for water distribution network optimization by using Genetic Algorithm. The program is written in MATLAB programming language. The program uses EPANET toolkit (a free open-source hydraulic solver) for steady state hydraulic simulation and solution. Before running the program, the network layout and network data must be imported as an .INP file from EPANET. Design parameters, such as available pipe sizes, respective cost of pipes, roughness coefficient, and required pressure and velocities for the network, should also be added. GA optimization parameters, such as population size, crossover probability, and mutation rate, are prerequisites to run the program, and are set to be altered by the user. Figure 1 shows a flowchart of the overall program:

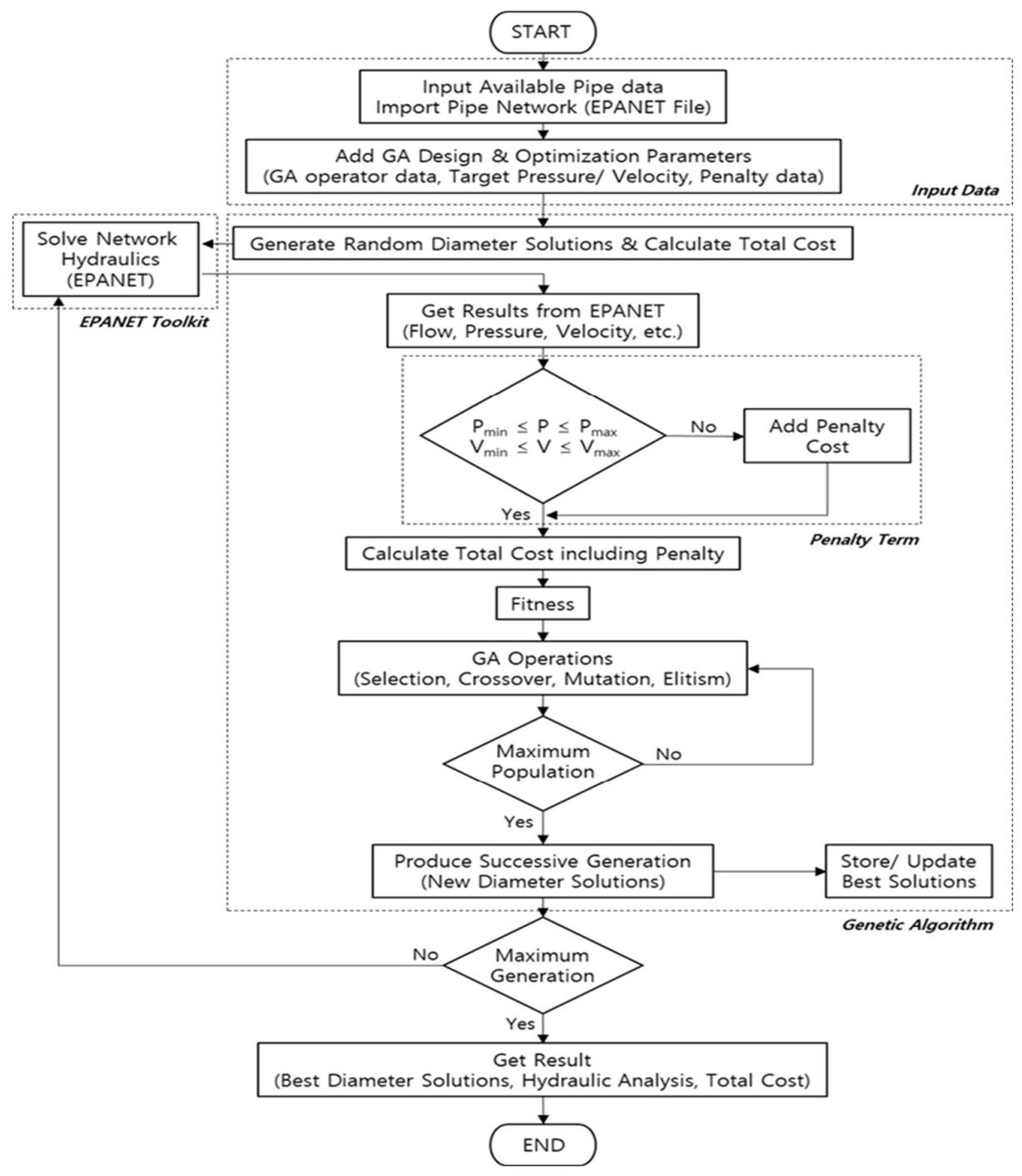

Figure 1. General Flowchart of the SOP-WDN program. 
The following Eqs. (5) \& (6) are used to calculate the penalty cost [28]. The penalty equation for violation of pressure constraint in the water distribution network implemented by SOP-WDN can be represented as:

$$
P_{P}=1+\sum_{j=1}^{N_{n}}\left|T_{P}-P_{j}\right| . P_{P 1}+\sum_{j=1}^{N_{n}}\left|T_{P}-P_{j}\right| \cdot P_{P 2}
$$

where, $N_{n}$ is the number of nodes in the network, $P_{P}$ the pressure penalty, $P_{j}$ the pressure of node $j, T_{P}$ the target pressure, $P_{P 1}$ the pressure penalty coefficient if the pressure at the node is above the target pressure, and $P_{P 2}$ the pressure penalty coefficient if the pressure at the node is below the target pressure.

The penalty equation for the violation of velocity constraint in the distribution network implemented by SOP-WDN can be represented as:

$$
V_{P}=1+\sum_{i=1}^{N_{p}}\left|T_{V}-V_{i}\right| . V_{P 1}+\sum_{i=1}^{N_{p}}\left|T_{V}-V_{i}\right| . V_{P 2}
$$

where, $N_{p}$ is the number of pipes in the network, $V_{P}$ the Velocity penalty, $V_{i}$ the flow velocity at link $i, T_{V}$ the target velocity, $V_{P 1}$ the velocity penalty coefficient if the velocity at a given link is above target velocity, and $V_{P 2}$ the velocity penalty coefficient if the velocity at the link is below target velocity.

The program computes the fitness of each member of the population as the reciprocal of the total cost (including penalty) with an exponent that is based on the number of pipes in the distribution network. The fitness values of all the population are then normalized, and a multiplier (based on average fitness) is utilized to multiply each of the normalized fitness values of the population.

\section{Results and discussion}

The conventional approach when testing the functionality, validity, and efficiency of a developed optimization program is to choose some benchmark WDN problems, and obtain their solution. Benchmark water distribution networks have provided a common testbed for newly developed optimization algorithms and design approaches. To prove their significance, the developed Metaheuristic Optimization Algorithms are applied to benchmark WDN problems and are compared to the existing algorithms. Using SOPWDN, some benchmark networks of the literature have been examined.

\subsection{Example 1: Two-loop network}

The two-loop network is an imaginary network introduced by Alperovitz and Shamir [3] that consists of 8 pipelines and 7 nodes (with reservoir), all fed by gravity flow from a single reservoir with an elevation of $210 \mathrm{~m}$. All pipes in the layout are 1,000 $\mathrm{m}$ in length, and the Hazen-Williams coefficient is 130. The minimum head requirement in each node is $30 \mathrm{~m}$ above ground level. The commercially available diameters and the details of the distribution network are described below:

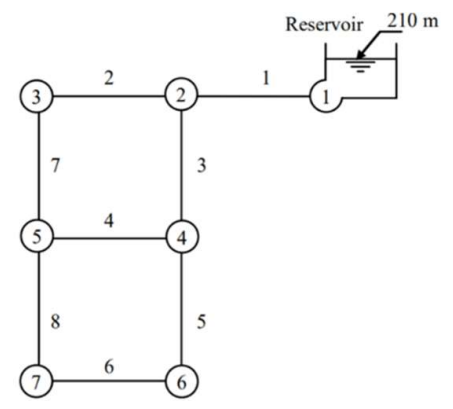

Figure 2. The Two-loop network layout. 
Table 1. Node data for the Two-loop network.

\begin{tabular}{ccc}
\hline Node No. & Elevation $(\mathbf{m})$ & Demand $\left(\mathbf{m}^{3} / \mathbf{h}\right)$ \\
\hline 1 & 210 & Reservoir \\
2 & 150 & 100 \\
3 & 160 & 100 \\
4 & 155 & 120 \\
5 & 150 & 270 \\
6 & 165 & 330 \\
7 & 160 & 200 \\
\hline
\end{tabular}

Table 2. Pipe data for the Two-loop network.

\begin{tabular}{cccc}
\hline Pipe No. & Begin node & End node & Length $(\mathbf{m})$ \\
\hline 1 & 1 & 2 & 1,000 \\
2 & 2 & 3 & 1,000 \\
3 & 2 & 4 & 1,000 \\
4 & 4 & 5 & 1,000 \\
5 & 4 & 6 & 1,000 \\
6 & 6 & 7 & 1,000 \\
7 & 3 & 5 & 1,000 \\
8 & 5 & 7 & 1,000 \\
\hline
\end{tabular}

Table 3. Available pipes for selection for the Two-loop network.

\begin{tabular}{ccc}
\hline Diameter (in) & Diameter $(\mathbf{m m})$ & Unit cost (USD/m) \\
\hline 1 & 25.4 & 2 \\
2 & 50.8 & 5 \\
4 & 101.6 & 11 \\
6 & 152.4 & 16 \\
10 & 254.0 & 32 \\
14 & 355.6 & 60 \\
16 & 406.4 & 90 \\
18 & 457.2 & 130 \\
\hline
\end{tabular}

Table 4 gives the solution obtained by SOP-WDN for the Two-loop network, while Fig. 3 shows the EPANET network layout of the solved network, and Table 5 compares the solution obtained from SOP-WDN with the solution obtained by other research reports:

Table 4. SOP-WDN results for the Two-loop network.

\begin{tabular}{cccc}
\hline Pipe No. & $\begin{array}{c}\text { Pipe diameter } \\
(\mathbf{m m})\end{array}$ & Pipe length $(\mathbf{m})$ & Cost (USD) \\
\hline 1 & 457.2 & 1,000 & 130,000 \\
2 & 254.0 & 1,000 & 32,000 \\
3 & 406.4 & 1,000 & 90,000 \\
4 & 101.6 & 1,000 & 11,000 \\
5 & 406.4 & 1,000 & 90,000 \\
\hline
\end{tabular}




\begin{tabular}{cccc}
\hline 6 & 254.0 & 1,000 & 32,000 \\
7 & 254.0 & 1,000 & 32,000 \\
8 & 25.4 & 1,000 & 2000 \\
\hline
\end{tabular}

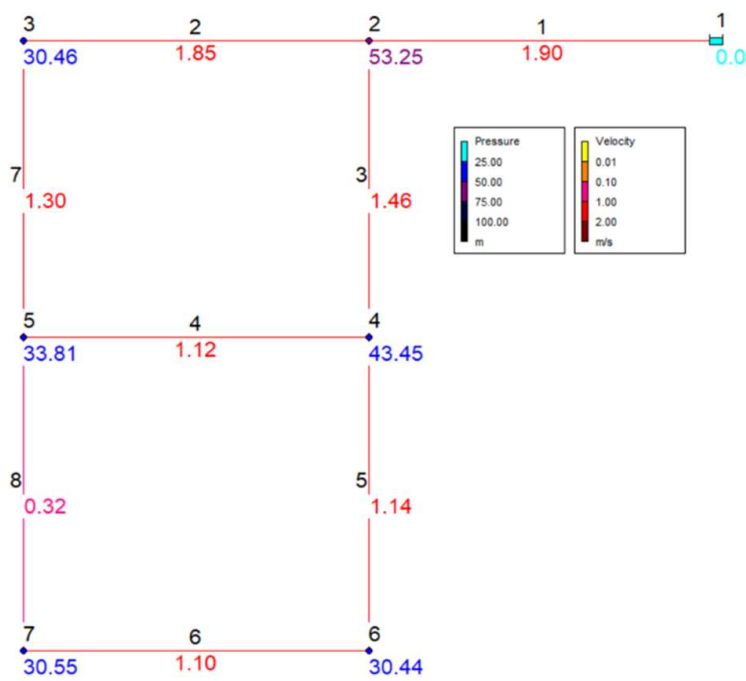

Figure 3. The Two-loop network solution (EPANET map) by SOP-WDN (pressure and velocity).

Table 5. Comparison of SOP-WDN results to past studies for the Two-loop network.

\begin{tabular}{cccccc}
\hline Studies & $\begin{array}{c}\text { Alperovitz } \\
\text { and Shamir }\end{array}$ & $\begin{array}{c}\text { Savic and } \\
\text { Walters }\end{array}$ & Geem & $\begin{array}{c}\text { Van Dijk et } \\
\text { al. }\end{array}$ & SOPWDN \\
\hline $\begin{array}{c}\text { Least cost } \\
\text { obtained } \\
\text { (USD) }\end{array}$ & 479,525 & 420,000 & 419,000 & 419,000 & 419,000 \\
\hline
\end{tabular}

The optimal cost of USD 419,000 was obtained, and the minimum pressure requirement of $30 \mathrm{~m}$ was fulfilled for all nodes. Table 5 shows the results obtained by other research reports for comparison. SOP-WDN obtained the optimal cost of USD 419,000 for the Two-loop network, which is same as the solution obtained by Geem and Van Dijk et al.

\subsection{Example 2: Hanoi network}

Hanoi network, located in Vietnam, was first presented by Fujiwara and Kang [29]. It consists of 32 nodes, 34 pipes, and 3 loops, and is fed by gravity from a reservoir with a $100 \mathrm{~m}$ fixed head. Table 7 shows the pipe lengths, which have a Hazen-Williams C of 130. The elevation of all nodes is $0 \mathrm{~m}$, and minimum head limitation is $30 \mathrm{~m}$ above ground level. The details of the distribution network are described below: 


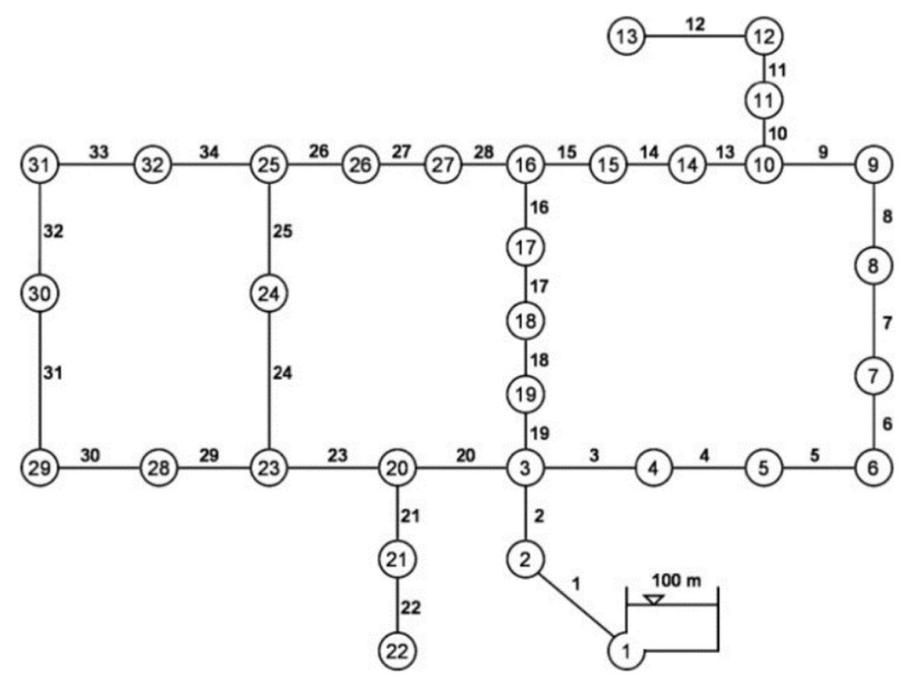

Figure 4. The Hanoi network layout.

Table 6. Node data for the Hanoi network.

\begin{tabular}{|c|c|}
\hline Node No. & Demand $\left(\mathrm{m}^{3} / \mathrm{h}\right)$ \\
\hline 1 & Reservoir \\
\hline 2 & 890 \\
\hline 3 & 850 \\
\hline 4 & 130 \\
\hline 5 & 725 \\
\hline 6 & 1,005 \\
\hline 7 & 1,350 \\
\hline 8 & 550 \\
\hline 9 & 525 \\
\hline 10 & 525 \\
\hline 11 & 500 \\
\hline 12 & 560 \\
\hline 13 & 940 \\
\hline 14 & 615 \\
\hline 15 & 280 \\
\hline 16 & 310 \\
\hline 17 & 865 \\
\hline 18 & 1,345 \\
\hline 19 & 60 \\
\hline 20 & 1,275 \\
\hline 21 & 930 \\
\hline 22 & 485 \\
\hline 23 & 1,045 \\
\hline 24 & 820 \\
\hline 25 & 170 \\
\hline 26 & 900 \\
\hline 27 & 370 \\
\hline 28 & 290 \\
\hline 29 & 360 \\
\hline 30 & 360 \\
\hline 31 & 105 \\
\hline
\end{tabular}




\section{2} 805

Table 7. Pipe data for the Hanoi network.

\begin{tabular}{|c|c|c|c|}
\hline Pipe No. & Begin node & End node & Length (m) \\
\hline 1 & 1 & 2 & 100 \\
\hline 2 & 2 & 3 & 1,350 \\
\hline 3 & 3 & 4 & 900 \\
\hline 4 & 4 & 5 & 1,150 \\
\hline 5 & 5 & 6 & 1,450 \\
\hline 6 & 6 & 7 & 450 \\
\hline 7 & 7 & 8 & 850 \\
\hline 8 & 8 & 9 & 850 \\
\hline 9 & 9 & 10 & 800 \\
\hline 10 & 10 & 11 & 950 \\
\hline 11 & 11 & 12 & 1,200 \\
\hline 12 & 12 & 13 & 3,500 \\
\hline 13 & 10 & 14 & 800 \\
\hline 14 & 14 & 15 & 500 \\
\hline 15 & 15 & 16 & 550 \\
\hline 16 & 17 & 16 & 2,730 \\
\hline 17 & 18 & 17 & 1,750 \\
\hline 18 & 19 & 18 & 800 \\
\hline 19 & 3 & 19 & 400 \\
\hline 20 & 3 & 20 & 2,200 \\
\hline 21 & 20 & 21 & 1,500 \\
\hline 22 & 21 & 22 & 500 \\
\hline 23 & 20 & 23 & 2,650 \\
\hline 24 & 23 & 24 & 1,230 \\
\hline 25 & 24 & 25 & 1,300 \\
\hline 26 & 26 & 25 & 850 \\
\hline 27 & 27 & 26 & 300 \\
\hline 28 & 16 & 27 & 750 \\
\hline 29 & 23 & 28 & 1,500 \\
\hline 30 & 28 & 29 & 2,000 \\
\hline 31 & 29 & 30 & 1,600 \\
\hline 32 & 30 & 31 & 150 \\
\hline 33 & 32 & 31 & 860 \\
\hline 34 & 25 & 32 & 950 \\
\hline
\end{tabular}

Table 8. Available pipes for selection for the Hanoi network.

\begin{tabular}{ccc}
\hline Diameter (in) & Diameter $(\mathbf{m m})$ & Unit cost $($ USD/m) \\
\hline 12 & 304.8 & 45.73 \\
16 & 406.4 & 70.40 \\
20 & 508 & 98.38 \\
24 & 609.6 & 129.33 \\
30 & 762 & 180.75 \\
40 & 1,016 & 278.28 \\
\hline
\end{tabular}




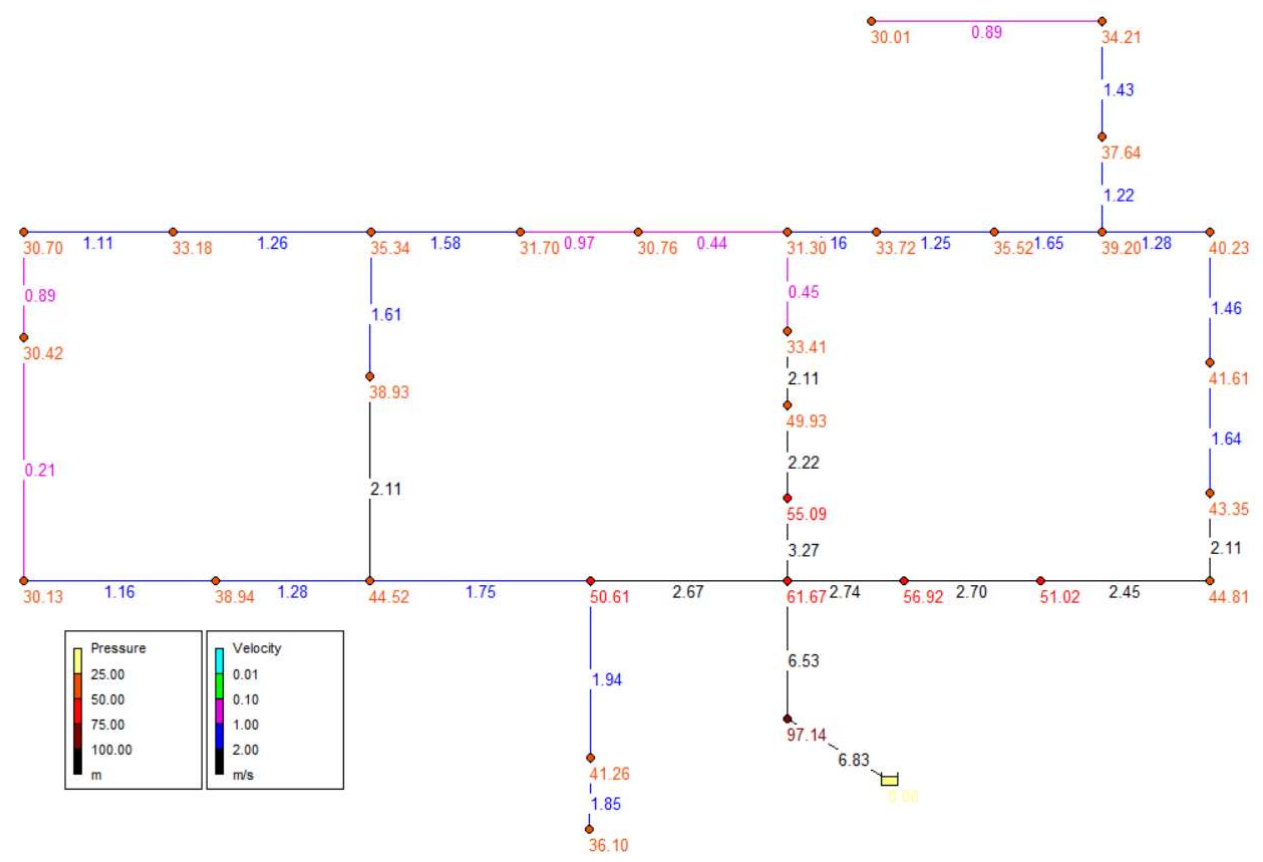

Figure 5. The Hanoi network solution (EPANET map) by SOP-WDN (pressure and velocity).

Table 9 gives the solution obtained by SOP-WDN for the Hanoi network, while Table 10 compares the solution obtained from SOP-WDN with the solution obtained by other research reports.

Table 9. SOP-WDN results for the Hanoi network.

\begin{tabular}{cccc}
\hline Pipe No. & $\begin{array}{c}\text { Pipe diameter } \\
(\mathbf{m m})\end{array}$ & Pipe length (m) & Cost (USD) \\
\hline 1 & 1,016 & 100 & 27,828 \\
2 & 1,016 & 1,350 & 375,678 \\
3 & 1,016 & 900 & 250,452 \\
4 & 1,016 & 1,150 & 320,022 \\
5 & 1,016 & 1,450 & 403,506 \\
6 & 1,016 & 450 & 125,226 \\
7 & 1,016 & 850 & 236,538 \\
8 & 1,016 & 850 & 236,538 \\
9 & 1,016 & 800 & 222,624 \\
10 & 762 & 950 & $171,712.5$ \\
11 & 609.6 & 1,200 & 155,196 \\
12 & 609.6 & 3,500 & 452,655 \\
13 & 508 & 800 & 78,704 \\
14 & 406.4 & 500 & 35,200 \\
15 & 304.8 & 550 & $25,151.5$ \\
16 & 304.8 & 2,730 & $124,842.9$ \\
17 & 406.4 & 1,750 & 123,200 \\
18 & 609.6 & 800 & 103,464 \\
19 & 508 & 400 & 39,352 \\
20 & 1,016 & 2,200 & 612,216 \\
21 & 508 & 1,500 & 147,570 \\
\hline
\end{tabular}




\begin{tabular}{cccc}
\hline 22 & 304.8 & 500 & 22,865 \\
23 & 1,016 & 2,650 & 737,442 \\
24 & 762 & 1,230 & $222,322.5$ \\
25 & 762 & 1,300 & 234,975 \\
26 & 508 & 850 & 83,623 \\
27 & 304.8 & 300 & 13,719 \\
28 & 304.8 & 750 & $34,297.5$ \\
29 & 406.4 & 1,500 & 105,600 \\
30 & 304.8 & 2,000 & 91,460 \\
31 & 304.8 & 1,600 & 73,168 \\
32 & 406.4 & 150 & 10,560 \\
33 & 406.4 & 860 & 60,544 \\
34 & 609.6 & 950 & $122,863.5$ \\
\hline
\end{tabular}

Table 10. Comparison of SOP-WDN results to past studies for the Hanoi network.

\begin{tabular}{cccccc}
\hline Studies & $\begin{array}{c}\text { Savic and } \\
\text { Walters }\end{array}$ & $\begin{array}{c}\text { Liong and } \\
\text { Atiquzzaman }\end{array}$ & Geem & $\begin{array}{c}\text { Van Dijk et } \\
\text { al. }\end{array}$ & SOPWDN \\
\hline $\begin{array}{c}\text { Least cost } \\
\text { obtained } \\
\text { (Million } \\
\text { USD) }\end{array}$ & 6.187 & 6.220 & 6.056 & 6.110 & 6.081 \\
\hline
\end{tabular}

The optimal cost of USD 6.081 million was obtained, and minimum pressure constraint of $30 \mathrm{~m}$ was fulfilled for all nodes. It was observed to be the best solution without the violation of any constraints.

\subsection{Example 3: GoYang network}

The GoYang water network is located in South Korea, and consists of 30 pipes, 22 nodes, and 9 loops. This network was first introduced by Kim et al. [30] and is fed by a single fixed pump of $4.52 \mathrm{~kW}$ from a $71 \mathrm{~m}$ constant head reservoir. The $\mathrm{H}-\mathrm{W}$ coefficient for all pipes in the network is 100 . The minimum head limitation for this network is $15 \mathrm{~m}$ above ground level. The commercially available diameters for the distribution network and the details of the distribution are described below:

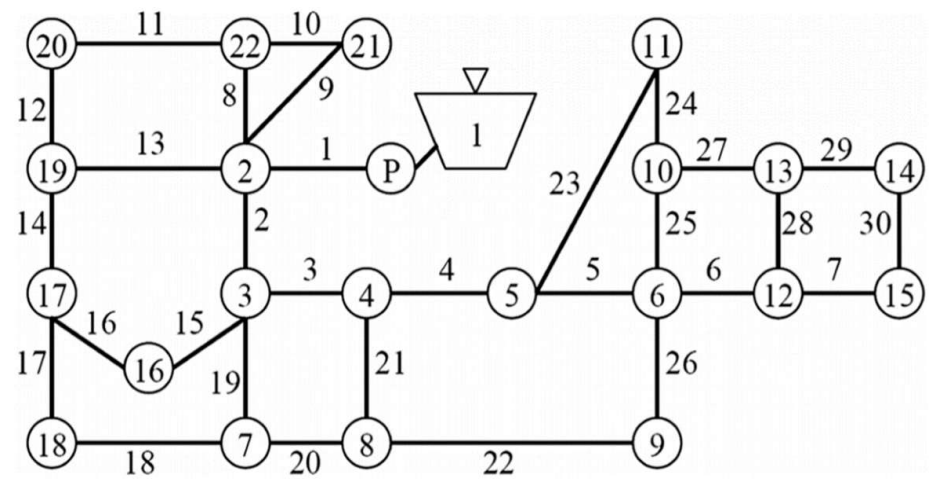

Figure 6. The GoYang network layout. 
Table 11. Node data for the GoYang network.

\begin{tabular}{ccc}
\hline Node No. & Elevation $(\mathbf{m})$ & Demand $\left(\mathbf{m}^{3} / \mathbf{d}\right)$ \\
\hline 1 & 71.0 & Reservoir \\
2 & 56.4 & 153.0 \\
3 & 53.8 & 70.5 \\
4 & 54.9 & 58.5 \\
5 & 56.0 & 75.0 \\
6 & 57.0 & 67.5 \\
7 & 53.9 & 63.0 \\
8 & 54.5 & 48.0 \\
9 & 57.9 & 42.0 \\
10 & 62.1 & 30.0 \\
11 & 62.8 & 42.0 \\
12 & 58.6 & 37.5 \\
13 & 59.3 & 37.5 \\
14 & 59.8 & 63.0 \\
15 & 59.2 & 445.5 \\
16 & 53.6 & 108.0 \\
17 & 54.8 & 79.5 \\
18 & 55.1 & 55.5 \\
19 & 54.2 & 118.5 \\
20 & 54.5 & 124.5 \\
21 & 62.9 & 31.5 \\
\hline
\end{tabular}

Table 12. Pipe data for the GoYang network.

\begin{tabular}{cccc}
\hline Pipe No. & Begin node & End node & Length $(\mathbf{m})$ \\
\hline 1 & 1 & 2 & 165 \\
2 & 2 & 3 & 124 \\
3 & 3 & 4 & 118 \\
4 & 4 & 5 & 81 \\
5 & 5 & 6 & 134 \\
6 & 6 & 12 & 135 \\
7 & 12 & 15 & 202 \\
8 & 2 & 22 & 135 \\
9 & 2 & 21 & 170 \\
10 & 21 & 22 & 113 \\
11 & 22 & 20 & 335 \\
12 & 20 & 19 & 115 \\
13 & 2 & 19 & 345 \\
14 & 19 & 17 & 114 \\
15 & 3 & 16 & 103 \\
16 & 16 & 17 & 261 \\
17 & 17 & 18 & 72 \\
18 & 7 & 18 & 373 \\
19 & 3 & 7 & 98 \\
20 & 7 & 8 & 110 \\
21 & 4 & 8 & 98 \\
22 & 8 & 9 & 246 \\
23 & 5 & 11 & 174 \\
\hline
\end{tabular}




\begin{tabular}{cccc}
\hline 24 & 10 & 11 & 102 \\
25 & 6 & 10 & 92 \\
26 & 6 & 9 & 100 \\
27 & 10 & 13 & 130 \\
28 & 12 & 13 & 90 \\
29 & 13 & 14 & 185 \\
30 & 15 & 14 & 90 \\
\hline
\end{tabular}

Table 13. Available pipes for selection for the GoYang network.

\begin{tabular}{cc}
\hline Diameter $(\mathbf{m m})$ & Unit cost $($ Won/m) \\
\hline 80 & 37,890 \\
100 & 38,933 \\
125 & 40,563 \\
150 & 42,554 \\
200 & 47,624 \\
250 & 54,125 \\
300 & 62,109 \\
350 & 71,524 \\
\hline
\end{tabular}

Table 14 gives the solution obtained by SOP-WDN for the GoYang network, while Fig. 7 shows the EPANET network layout of the solved network. Table 15 compares the solution obtained from SOP-WDN with the solution obtained by other research reports.

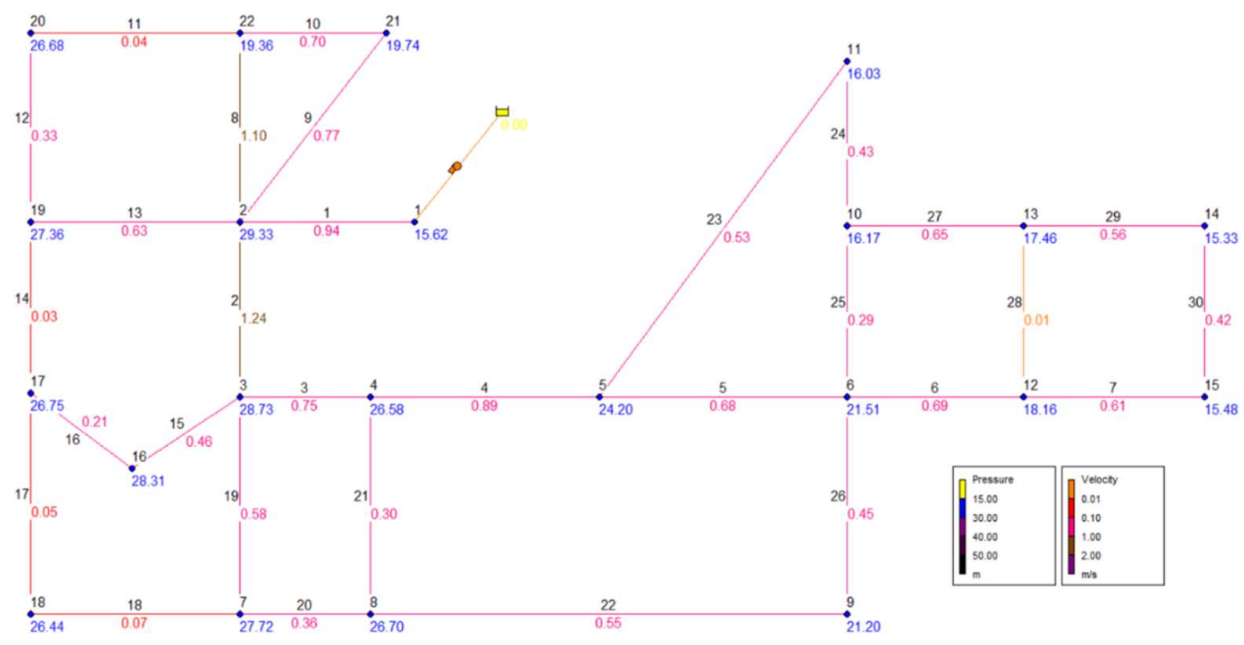

Figure 7. The GoYang network solution (EPANET map) by SOP-WDN (pressure and velocity).

Table 14. SOP-WDN results for the GoYang network.

\begin{tabular}{cccc}
\hline Pipe No. & $\begin{array}{c}\text { Pipe diameter } \\
(\mathbf{m m})\end{array}$ & Pipe length $(\mathbf{m})$ & Cost (Won) \\
\hline 1 & 200 & 165.0 & $7,857,960$ \\
2 & 125 & 124.0 & $5,029,812$ \\
3 & 125 & 118.0 & $4,786,434$ \\
4 & 100 & 81.0 & $3,153,573$ \\
\hline
\end{tabular}




\begin{tabular}{|c|c|c|c|}
\hline 5 & 80 & 134.0 & $5,077,260$ \\
\hline 6 & 80 & 135.0 & $5,115,150$ \\
\hline 7 & 80 & 202.0 & $7,653,780$ \\
\hline 8 & 80 & 135.0 & $5,115,150$ \\
\hline 9 & 80 & 170.0 & $6,441,300$ \\
\hline 10 & 80 & 113.0 & $4,281,570$ \\
\hline 11 & 80 & 335.0 & $12,693,150$ \\
\hline 12 & 80 & 115.0 & $4,357,350$ \\
\hline 13 & 80 & 345.0 & $13,072,050$ \\
\hline 14 & 80 & 114.0 & $4,319,460$ \\
\hline 15 & 80 & 103.0 & $3,902,670$ \\
\hline 16 & 80 & 261.0 & $9,889,290$ \\
\hline 17 & 80 & 72.0 & $2,728,080$ \\
\hline 18 & 80 & 373.0 & $14,132,970$ \\
\hline 19 & 80 & 98.0 & $3,713,220$ \\
\hline 20 & 80 & 110.0 & $4,167,900$ \\
\hline 21 & 80 & 98.0 & $3,713,220$ \\
\hline 22 & 80 & 246.0 & $9,320,940$ \\
\hline 23 & 80 & 174.0 & $6,592,860$ \\
\hline 24 & 80 & 102.0 & $3,864,780$ \\
\hline 25 & 80 & 92.0 & $3,485,880$ \\
\hline 26 & 80 & 100.0 & $3,789,000$ \\
\hline 27 & 80 & 130.0 & $4,925,700$ \\
\hline 28 & 80 & 90.0 & $3,410,100$ \\
\hline 29 & 80 & 185.0 & $7,009,650$ \\
\hline \multirow[t]{2}{*}{30} & 80 & 90.0 & $3,410,100$ \\
\hline & & Total & $177,010,359$ \\
\hline
\end{tabular}

Table 15. Comparison of the SOP-WDN results to those of past studies of the GoYang network.

\begin{tabular}{cccccc}
\hline Studies & $\begin{array}{c}\text { Original } \\
\text { Network }\end{array}$ & Kim et al. & Geem & Menon et al. & SOPWDN \\
\hline $\begin{array}{c}\text { Least cost } \\
\text { obtained } \\
\text { (Million } \\
\text { Won) }\end{array}$ & 179.428 & 179.142 & 177.135 & 177.417 & 177.010 \\
\hline
\end{tabular}

The lowest cost obtained by SOP-WDN was 177,010,359 Won, which compared to the other studies, is the cheapest cost. The obtained solution also has no nodes containing pressure violations, as all nodes in the distribution network have fulfilled the minimum pressure requirement of $15 \mathrm{~m}$.

\section{Conclusions}

In this study, the developed Genetic Algorithm based optimization program SOPWDN was tested on three benchmark water distribution networks, and in comparison to the other studies, it was able to produce competitive results. EPANET software, which was used for the hydraulic analysis and calculations of the water distribution systems is a well-accepted and utilized software. EPANET-MATLAB toolkit enabled the SOP-WDN program to perform EPANET based calculations directly in the MATLAB environment, which improved the overall speed, performance, and efficiency of the program. Hence, 
the EPANET-MATLAB toolkit can prove to be an important tool that enables the facile use of EPANET software in the MATLAB environment for many different research purposes. SOP-WDN can be used as a reliable program that can easily be implemented and adapted to aid engineers and designers during the design process of new water distribution networks, or the rehabilitation of existing water distribution networks.

Supplementary Materials: Not applicable.

Author Contributions: Uchit Sangroula developed the SOP-WDN program, and wrote the manuscript of this study; Kuk-Heon Han, Kang-Min Koo, and Kapil Gnawali made recommendations during the writing of the manuscript; Kyung-Taek Yum provided significant suggestions on the methodology and structure of the manuscript. All authors have read and approved the final manuscript.

Funding: This Research has been performed as Project of No A-C-002 supported by K-water.

Institutional Review Board Statement: Not applicable.

Informed Consent Statement: Not applicable.

Data Availability Statement: Datasets that are restricted and not publicly available.

Conflicts of Interest: The authors declare no conflict of interest.

\section{References}

1. Swamee, P.K.; Sharma, A.K. Design of water supply pipe networks. John Wiley \& Sons, 2008.

2. Cross, H. Analysis of flow in networks of conduits or conductors. University of Illinois at Urbana Champaign, College of Engineering. Engineering Experiment Station. 1936.

3. Alperovits, E.; Shamir, U. Design of optimal water distribution systems. Water resources research 1977, 13(6), 885-900.

4. Goulter, I.C.; Lussier, B.M.; Morgan, D.R. Implications of head loss path choice in the optimization of water distribution networks. Water Resources Research 1986, 22(5), 819-822.

5. Quindry, G.E.; Liebman, J.C.; Brill, E.D. Optimization of looped water distribution systems. Journal of the Environmental Engineering Division 1981, 107(4), 665-679.

6. Goldberg, D.E.; Kuo, C.H. Genetic algorithms in pipeline optimization. Journal of Computing in Civil Engineering 1987, 1(2), 128141.

7. Simpson, A.; Murphy, L.; Dandy, G. Pipe network optimisation using genetic algorithms. American Society of Civil Engineers 1993.

8. Simpson, A.R.; Dandy, G.C.; Murphy, L.J. Genetic algorithms compared to other techniques for pipe optimization. Journal of water resources planning and management 1994, 120(4), 423-443.

9. Dandy, G.C.; Simpson, A.R.; Murphy, L.J. An improved genetic algorithm for pipe network optimization. Water resources research 1996, 32(2), 449-458.

10. Savic, D.A.; Walters, G.A. Genetic algorithms for least-cost design of water distribution networks. Journal of water resources planning and management 1997, 123(2), 67-77.

11. Deb, K.; Agrawal, S. A niched-penalty approach for constraint handling in genetic algorithms. Artificial Neural Nets and Genetic Algorithms 1996, 235-243.

12. Wu, Z.Y.; Simpson, A.R. A self-adaptive boundary search genetic algorithm and its application to water distribution systems. Journal of hydraulic research 2002, 40(2), 191-203.

13. Liong, S.Y.; Atiquzzaman, M. Optimal design of water distribution network using shuffled complex evolution. Journal of The Institution of Engineers, Singapore 2004, 44(1), 93-107.

14. Eusuff, M.; Lansey, K.; Pasha, F. Shuffled frog-leaping algorithm: a memetic meta-heuristic for discrete optimization. Engineering optimization 2006, 38(2), 129-154.

15. Geem, Z.W. Optimal cost design of water distribution networks using harmony search. Engineering optimization 2006, 38(03), 259-277.

16. Di Pierro, F.; Khu, S. T.; Savić, D.; Berardi, L. Efficient multi-objective optimal design of water distribution networks on a budget of simulations using hybrid algorithms. Environmental Modelling \& Software 2009, 24(2), 202-213. 
17. Chandramouli, S.; Malleswararao, P. Reliability based optimal design of a water distribution network for municipal water supply. International journal of engineering and technology 2011, 3(1), 13-19.

18. Jin, X.; Zhang, J.; Gao, J. L.; Wu, W. Y. Multi-objective optimization of water supply network rehabilitation with non-dominated sorting genetic algorithm-II. Journal of Zhejiang University-SCIENCE A 2008, 9(3), 391-400.

19. Kang, D.; Lansey, K. Revisiting optimal water-distribution system design: issues and a heuristic hierarchical approach. Journal of Water resources planning and management 2012, 138(3), 208-217.

20. Cisty, M.; Bajtek, Z.; Celar, L. A two-stage evolutionary optimization approach for an irrigation system design. Journal of Hydroinformatics 2017, 19(1), 115-122.

21. Van Dijk, M.; van Vuuren, S. J.; Van Zyl, J. E. Optimising water distribution systems using a weighted penalty in a genetic algorithm. Water $S A$ 2008, 34(5), 537-548.

22. Lansey, K. E.; Mays, L. W. Optimization models for design of water distribution systems. Reliability Analysis of Water Distribution Systems 1989. 37-84.

23. Keedwell, E.; Khu, S. T. A hybrid genetic algorithm for the design of water distribution networks. Engineering Applications of Artificial Intelligence 2005, 18(4), 461-472.

24. Goldberg, D. E. A Gentle Introduction to Genetic Algorithms. Addison-Wesley Publishing Company, 1989.

25. Abuiziah, I.; Nidal, S. A review of genetic algorithm optimization: operations and applications to water pipeline systems, 2013.

26. Rossman, L. A. EPANET 2: user's manual, 2000

27. Eliades, D. G.; Kyriakou, M.; Vrachimis, S.; Polycarpou, M. M. EPANET-MATLAB toolkit: An open-source software for interfacing EPANET with MATLAB. Proceedings of the 14th International Conference on Computing and Control for the Water Industry 2016.

28. Güç, G. Optimization of water distribution networks using genetic algorithm. Master's thesis, Middle East Technical Univ, 2006.

29. Fujiwara, O.; Kang, D. B. A two-phase decomposition method for optimal design of looped water distribution networks. Water Resources Research 1990, 26(4), 539-549.

30. Kim, J. H.; Kim, T. G.; Kim, J. H.; Yoon, Y. N. A study on the pipe network system design using non-linear programming. J Korean Water Resource Association 1994, 27(4), 59-67.

31. Menon, K. K; Narulkar, S. M. Application of heuristic based algorithm in water distribution network design Journal of Water Resource Pollution Study 2016, 1-14. 